\title{
Soporte circulatorio con ECMO venoarterial en paciente con cardiomiopatía de Takotsubo biventricular
}

\author{
Circulatory support with venoarterial ECMO in a patient with biventricular Takotsubo \\ cardiomyopathy
}

\author{
Emmanuel A. Lazcano-Díaz ${ }^{*}$, Francisco J. González-Ruíz², Diego Sarre-Álvarez ${ }^{3}$, Rolando J. Álvarez-Álvarez², \\ Eduardo Bucio-Reta², Edgar García-Cruz², Gustavo Rojas-Velasco², Ángel Ramos-Enríquez², \\ Efrén Melano-Carranza², Luis E. Santos-Martínez ${ }^{2}$ y Francisco M. Baranda-Tovar ${ }^{4}$ \\ ${ }^{1}$ Servicio de Terapia Intensiva Cardiovascular; ${ }^{2}$ Servicio de Terapia Posquirúrgica; ${ }^{3}$ Servicio de Cardiología; ${ }^{4}$ Subdirección. Instituto Nacional de \\ Cardiología Ignacio Chávez de México, Ciudad de México, México
}

\begin{abstract}
Resumen
La cardiomiopatía de Takotsubo es una entidad caracterizada por disfunción ventricular aguda y transitoria, la cual está generalmente relacionada a un evento desencadenante (estrés emocional o físico) y que, por lo general, se presenta con disfunción sistólica regional del ventrículo izquierdo, aunque hasta en un 30\% puede ser biventricular. Según su severidad, en algunos casos puede condicionar choque cardiogénico refractario a manejo con inotrópicos y vasopresores, por lo que para estos casos deben considerarse los dispositivos de asistencia circulatoria. Presentamos el caso de una paciente joven a quien se realizó cambio valvular pulmonar con prótesis biológica, la cual siete semanas posteriores a la cirugía acudió al servicio de urgencias con derrame pericárdico y fisiología de tamponade secundario a síndrome pospericardiotomía. Por tal motivo se le practicó ventana pericárdica, sin embargo durante el transquirúrgico presentó cardiomiopatía de Takotsubo biventricular que le condicionó choque cardiogénico con insuficiencia mitral y tricúspidea severas y refractariedad a tratamiento médico, así como a balón intraaórtico de contrapulsación (BIAC), por lo cual requirió soporte circulatorio con ECMO venoarterial durante 5 días.
\end{abstract}

Palabras clave: Cardiomiopatía de Takotsubo. Disfunción ventricular. Choque cardiogénico. ECMO venoarterial.

\begin{abstract}
Takotsubo cardiomyopathy is an entity characterized by acute and transient ventricular dysfunction, which is usually related to a triggering event (emotional or physical stress), and usually presents with regional systolic dysfunction of the left ventricle, however up to $30 \%$ may be biventricular. Depending on its severity in some cases the disease can condition refractory cardiogenic shock to management with inotropics and vasopressors, so for these cases circulatory assistance devices should be considered. We present the case of a young patient who had pulmonary valve change with biological prosthesis, which seven weeks after surgery went to the emergency department with pericardial effusion and tamponade physiology secondary
\end{abstract}

\footnotetext{
Correspondencia:

Fecha de recepción: 05-02-2020

Disponible en internet: 05-08-2020

*Emmanuel A. Lazcano-Díaz

Fecha de aceptación: 10-05-2020

E-mail: ea.lazcano.diaz@gmail.com

DOI: $10.24875 /$ ACM.20000038

Arch Cardiol Mex. 2021;91(1):100-104 www.archivoscardiologia.com

1405-9940 / @ 2020 Instituto Nacional de Cardiología Ignacio Chávez. Publicado por Permanyer. Este es un artículo open access bajo la licencia CC BY-NC-ND (http://creativecommons.org/licenses/by-nc-nd/4.0/).
} 
to postpericardiotomy syndrome. For this reason pericardial window was practiced, however during the procedure she presented biventricular Takotsubo cardiomyopathy which conditioned cardiogenic shock with severe mitral and tricuspid regurgitation, and refractivity to medical treatment as well as intraaortic balloon pump, requiring circulatory support with venoarterial ECMO for 5 days.

Key words: Takotsubo cardiomyopathy. Ventricular dysfunction. Cardiogenic shock. Venoarterial ECMO.

Se presenta el caso de una paciente de 37 años de edad con antecedente de doble lesión pulmonar (predominio de estenosis) a quien se le realizó cambio valvular pulmonar por prótesis biológica sin complicaciones perioperatorias. Egresada a domicilio asintomática con función biventricular conservada. Sin embargo, seis semanas después de procedimiento quirúrgico cursó con disnea de medianos a pequeños esfuerzos, edema de miembros pélvicos ascendente y dolor tipo pleurítico, razón por la cual acudió al servicio de urgencias, en donde ingresó con tendencia a hipotensión, frote pericárdico, edema importante de extremidades, electrocardiograma con taquicardia sinusal, complejos QRS con bajo voltaje y alternancia eléctrica. La radiografía de tórax y el ultrasonido pulmonar, con derrame pleural bilateral importante. Laboratorios relevantes con: porción $\mathrm{N}$-terminal del propéptido natriurético tipo $\mathrm{B}$ (NT-proBNP) $2,305 \mathrm{pg} / \mathrm{ml}$, troponina de alta sensibilidad 2,3 $\mathrm{ng} / \mathrm{ml}$ y proteína C reactiva (PCR) $121 \mathrm{mg} / \mathrm{l}$. Se realizó ecocardiograma dirigido, con reporte de derrame pericárdico global de $49 \mathrm{~mm}$ con variabilidad transmitral en el 53\%, transtricuspídea del 39\%, colapso de aurícula derecha y función biventricular conservada sin trastornos de la movilidad. Ante repercusión hemodinámica se decidió realizar ventana pericárdica drenando $1,000 \mathrm{cc}$ de líquido seroso, así como colocación de sondas endopleurales drenando 1,200 cc de líquido seroso, ambos con reporte citoquímico de exudado de características inflamatorias con cultivo negativo. Posterior a ventana pericárdica fue trasladada a terapia posquirúrgica, en donde se recibió hipotensa con datos de hipoperfusión sistémica. Se realizó nuevo electrocardiograma, que evidenció alargamiento del intervalo QTc, así como inversión de ondas T en cara anterior (Fig. 1); ecocardiograma que mostró función biventricular deprimida, ventrículo izquierdo con acinesia en región media y apical con hipercinesia compensatoria del tercio basal en todas sus caras; fracción de expulsión del ventrículo izquierdo (FEVI) en el 15\% por Simpson modificado, strain -3\%, (Fig. 2), FEVI tridimensional en el $20 \%$; ventrículo derecho con acinesia en tercios basal y apical, e hipercinesia basal, así mismo con fracción de acortamiento del ventrículo derecho (FACVD) del 15\%, sugestivo de cardiomiopatía de Takotsubo biventricular sin obstrucción del tracto de salida del ventrículo izquierdo (Fig. 3). Así mismo, se reportó insuficiencia mitral y tricuspídeas severas asociadas a tethering (Figs. 4 y 5). Se realizó nueva medición de troponinas con elevación hasta $3,200 \mathrm{ng} / \mathrm{ml}$ y NT-proBNP 4,000 pg/ml. En cuanto al perfil hemodinámico, en choque cardiogénico, por lo que se inició manejo con doble inotrópico (levosimendán y dobutamina) y doble vasopresor (norepinefrina y vasopresina); sin embargo persistió con índice cardiaco en $1.4 \mathrm{l} / \mathrm{min}$ y datos de hipoperfusión sistémica, por lo que se decidió colocar balón de contrapulsación intraaórtica (BIAC). A pesar de la terapéutica empleada la paciente continuó en choque cardiogénico refractario con compromiso respiratorio requiriendo intubación orotraqueal y presentando disfunción hepática y renal, por lo que 12 horas posteriores a inicio de terapia con BIAC se agregó soporte con membrana de oxigenación extracorpórea (ECMO) por canulación femoral venosa y femoral arterial, titulando el flujo hasta $2.5 \mathrm{l} / \mathrm{min}$. Durante el soporte circulatorio cursó con adecuada evolución, con estabilización hemodinámica, lográndose retirar las aminas e inotrópicos en las primeras 4 horas, con posterior mejoría de función renal y hepática, así como disminución de la congestión pulmonar y logrando extubación en las primeras 48 horas. En cuanto a la función biventricular, con clara mejoría progresiva (hasta lograr FEVI del 35\%, integral tiempo velocidad (ITV) $14 \mathrm{~cm}$, velocidad «s» mitral lateral $8 \mathrm{~cm} / \mathrm{s}$ y FACVD del $30 \%$ ), con remisión de las alteraciones segmentarias de la contractilidad y disminución en la severidad de las valvulopatías (hasta ser de grado ligero), sin complicaciones relacionadas a la ECMO, por lo que se suspendió la terapia a los cinco días de manera exitosa.

Posteriormente se trasladó a piso de cardiología, en donde se realizó resonancia magnética cardiaca, reportándose sin trastornos de la movilidad, función biventricular conservada, sin insuficiencia valvulares y con bioprótesis pulmonar normofuncionante. 


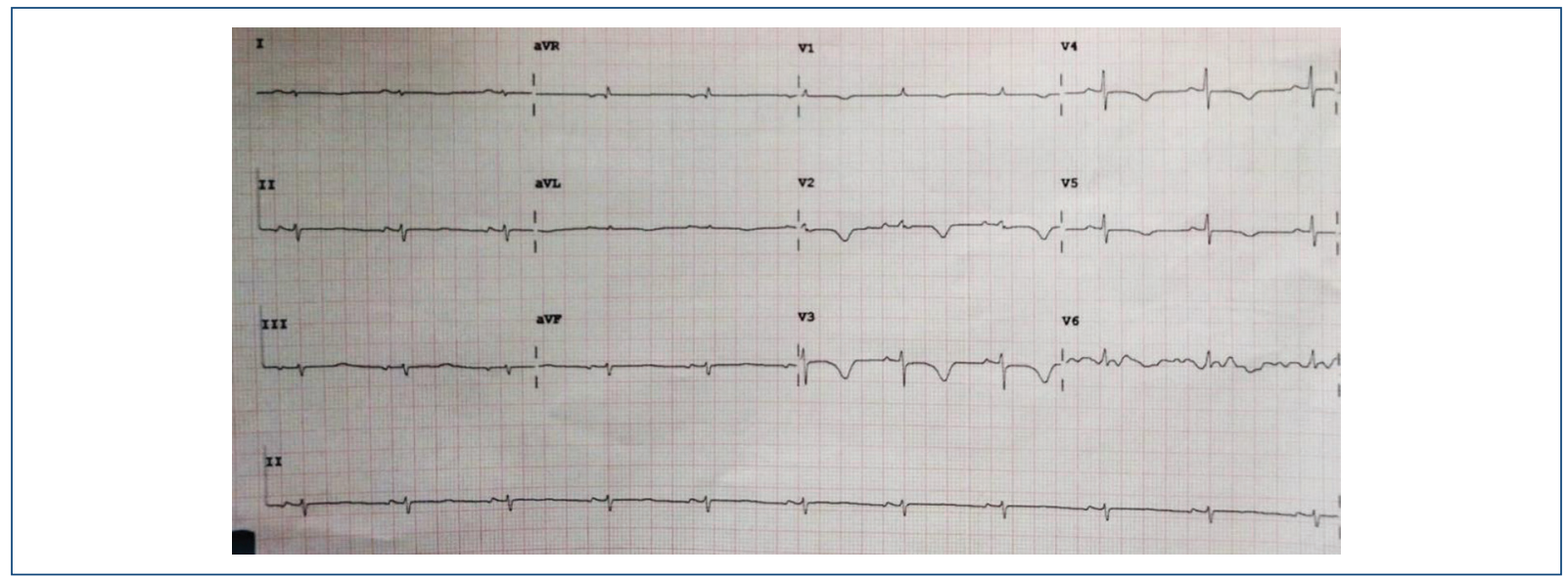

Figura 1. Electrocardiograma de la paciente posterior a ventana pericárdica con hallazgos sugerentes de cardiomiopatía de Takotsubo.

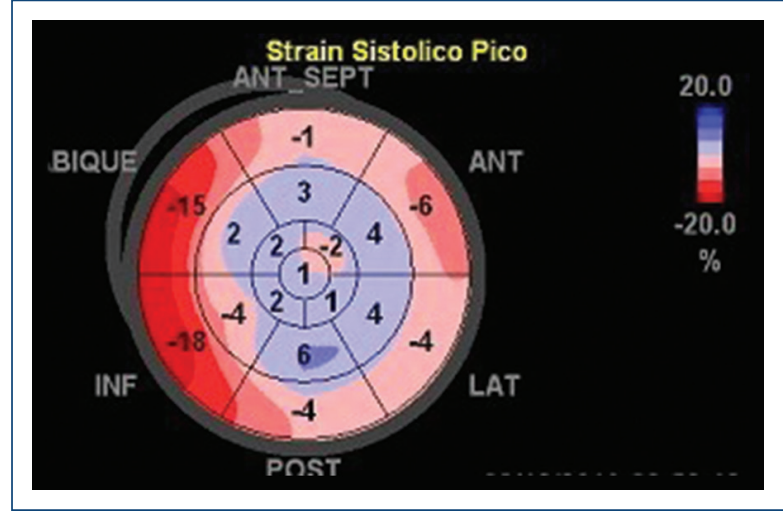

Figura 2. Strain de ventrículo izquierdo posterior a ventana pericárdica con hallazgos sugerentes de cardiomiopatía de Takotsubo.

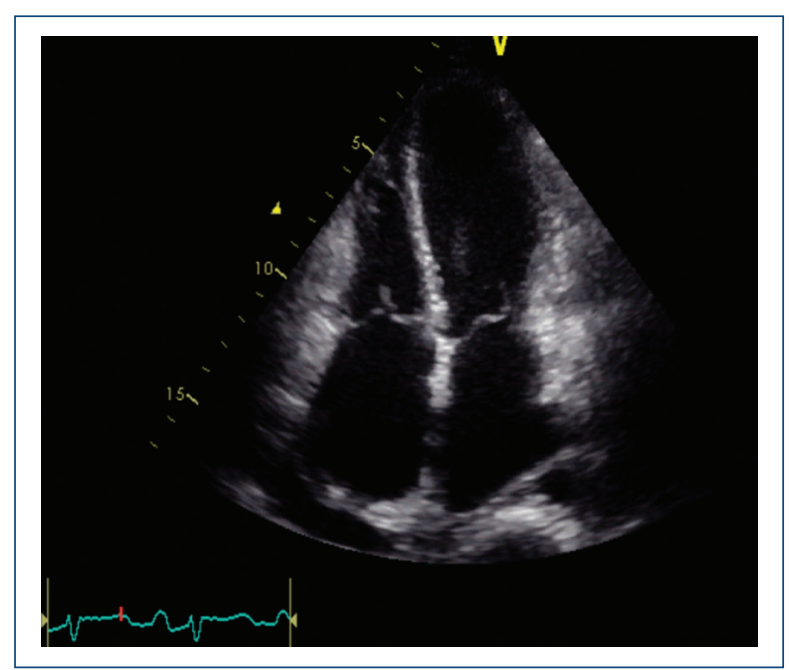

Figura 3. Aproximación apical de cuatro cámaras en donde se observan los hallazgos sugerentes de cardiomiopatía de Takotsubo biventricular.

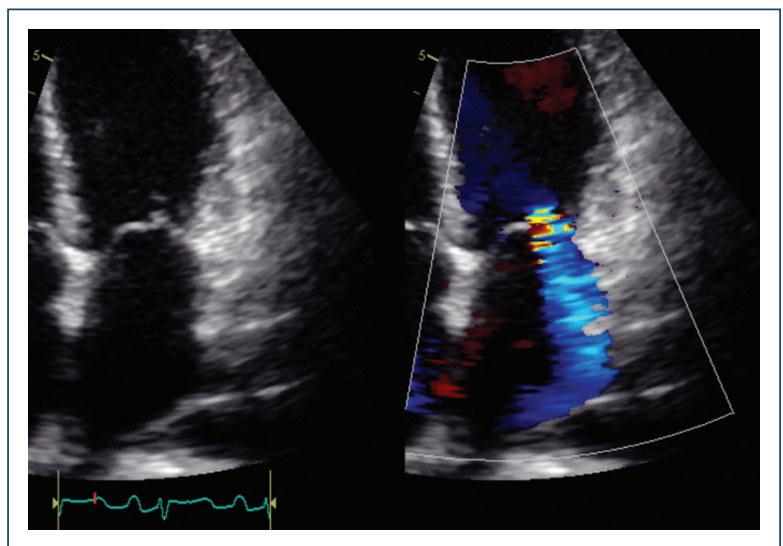

Figura 4. Aproximación apical de cuatro cámaras en donde se observa insuficiencia valvular mitral secundaria a tethering.

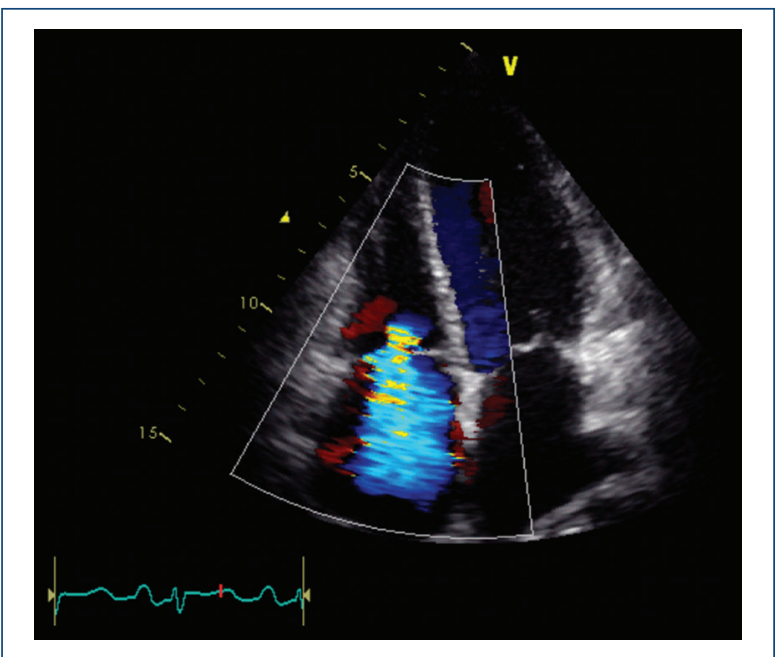

Figura 5. Aproximación apical de cuatro cámaras en donde se observa insuficiencia valvular tricuspídea secundaria a tethering. 


\section{Revisión de la literatura}

Se presenta el caso de una paciente que siete semanas después de cirugía cardiaca presentó síndrome pospericardiotomía, el cual según la bibliografía tiene una prevalencia de hasta un $10-40 \%$ y se puede presentar desde los primeros días hasta varias semanas posteriores a la cirugía. Clínicamente este síndrome se presenta hasta en el $80 \%$ de la veces con dolor torácico, en el $50-60 \%$ con febrícula y en el $50-60 \%$ con disnea; pueden existir derrames tanto en pericardio (> $80 \%$ ) como en pleura (> 60\%) y frote pericárdico hasta en el $30-60 \%$ de los pacientes. Los análisis de laboratorio muestran elevación de la PCR (74\%) y leucocitosis ${ }^{1-3}$.

En nuestro caso la paciente se presentó a urgencias con tamponade e inestabilidad hemodinámica, por lo cual se decidió realizar ventana pericárdica urgente. Es bien conocido que el síndrome de Takotsubo es secundario a una elevación de catecolaminas séricas y que el estrés físico o emocional pueden desencadenar el cuadro. En este caso tanto el tamponade como el mismo procedimiento quirúrgico pudieron actuar como desencadenantes para desarrollar la cardiomiopatía de estrés.

El electrocardiograma y el ecocardiograma posteriores a la ventana pericárdica mostraron hallazgos bien descritos previamente en nuestra paciente, y que se pueden encontrar en la literatura actual de miocardiopatía de Takotsubo tipo I (InterTAK) ${ }^{4}$. Adicionalmente se ha evidenciado la obstrucción del tracto de salida del ventrículo izquierdo hasta en el $20 \%$ de los casos, lo que condiciona insuficiencia mitral en las fases agudas. Es de notable importancia recalcar que en nuestro paciente se presentó insuficiencia mitral no asociada con obstrucción del tracto de salida del ventrículo izquierdo (gradiente medio de $5 \mathrm{mmHg}$ ), pero sí a las alteraciones de la movilidad segmentaria que limitan el cierre de la valva posterior (tethering de la valva/insuficiencia mitral IIIB de Carpentier), asociadas con mal posición del aparato valvular mitral. Así mismo, la presentación de cardiomiopatía de Takotsubo con involucro del ventrículo derecho no es infrecuente (se reporta una incidencia del $34 \%)^{5}$. Los hallazgos característicos del Takotsubo que involucran el ventrículo derecho están relacionados con la dilatación de la cavidad y alteraciones de la pared lateral, alteraciones presentes en este caso con hipercinesia basal y alteraciones en la movilidad del segmento apical.

El método auxiliar no invasivo de elección para el diagnóstico es el ecocardiograma, ya que permite apreciar el patrón distintivo de la disfunción sistólica y realizar evaluaciones subsecuentes para demostrar la remisión, así como identificar los casos con peor pronóstico (aquellos con insuficiencia valvular por movimiento sistólico anterior de la valva mitral anterior o con obstrucción del tracto de salida del ventrículo izquierdo por hipercontractilidad basal, condición que confiere dificultad al manejo de la falla cardiaca con inotrópicos, ya que estos pueden empeorar la obstrucción).

El tratamiento se centra en el manejo de la falla cardiaca. En caso de hipotensión y shock se deberá considerar el uso de inotrópicos como estrategia para aumentar el gasto cardiaco. Si estos no son suficientes se deberá valorar agregar vasopresores a dosis bajas y por el menor tiempo posible como puente a la mejoría o puente al soporte circulatorio con balón de contra pulsación aórtica o ECMO venoarterial ${ }^{6}$.

Hasta un $20 \%$ de los pacientes con cardiomiopatía de Takotsubo presentan obstrucción del tracto de salida del ventrículo izquierdo, lo cual genera mayor disminución del gasto cardiaco (ya de por sí deteriorado por la disfunción sistólica), por lo que entender su fisiopatología es trascendental para el manejo. Toda maniobra que favorezca la contracción y colapso ventricular favorecerá el gradiente obstructivo, por lo que se debe evitar el uso de inotrópicos, mientras que las maniobras que favorecen el llenado ventricular (como aumentar la precarga con soluciones) son benéficas. El uso de betabloqueadores como el esmolol o el metoprolol también puede disminuir el gradiente al disminuir la contracción miocárdica. Por último, en casos refractarios a estas medidas se debe considerar el uso de dispositivos de asistencia mecánica, preferiblemente asistencia con $\mathrm{ECMO}^{7,8}$.

\section{Agradecimientos}

Al Instituto Nacional de Cardiología Ignacio Chávez de México.

\section{Financiamiento}

La presente investigación no ha recibido ayudas específicas provenientes de agencias del sector público, sector comercial o entidades sin ánimo de lucro.

\section{Conflicto de intereses}

Los autores declaran no tener conflicto de intereses. 


\section{Responsabilidades éticas}

Protección de personas y animales. Los autores declaran que para esta investigación no se han realizado experimentos en seres humanos ni en animales.

Confidencialidad de los datos. Los autores declaran que en este artículo no aparecen datos de pacientes.

Derecho a la privacidad y consentimiento informado. Los autores declaran que en este artículo no aparecen datos de pacientes.

\section{Bibliografía}

1. Finkelstein Y, Shemesh J, Mahlab K, Abramov D, Bar-El Y, Sagie A et al. Colchicine for the prevention of postpericardiotomy syndrome. Herz. 2002;27(8):791-4
2. Imazio M, Trinchero R, Brucato A, Rovere ME, Gandino A, Cemin R, et al.; COPPS Investigators. COlchicine for the Prevention of the Post-pericardiotomy Syndrome (COPPS): a multicentre, randomized, double-blind, placebo-controlled trial. Eur Heart J. 2010;31(22):2749-54.

3. Imazio M, Brucato A, Ferrazzi P, Pullara A, Adler $Y$, Barosi $A$, et al.; COPPS-2 Investigators. Colchicine for Prevention of postpericardiotomy syndrome and postoperative atrial fibrillation: the COPPS-2 randomized clinical trial. JAMA. 2014;312(10):1016-23.

4. Ghadri JR, Wittstein IS, Prasad A, Sharkey S, Dote K, Akashi YJ, et al. International expert consensus document on Takotsubo syndrome (Part I): Clinical characteristics, diagnostic criteria, and pathophysiology. Eur Heart J. 2018;39(22):2032凸46.

5. Eitel I, von Knobelsdorff F, Bernhardt P, Carbone I, Muellerleile K, Aldrovandi $A$, et al. Clinical characteristics and cardiovascular magnetic resonance findings in stress (Takotsubo) cardiomyopathy. JAMA. 2011;306:277-86.

6. Rashed A, Won S, Saad M, Schreiber T. Use of the Impella 2.5 left ventricular assist device in a patient with cardiogenic shock secondary to Takotsubo cardiomyopathy. BMJ Case Rep. 2015;2015:1-4.

7. Yoshioka T, Hashimoto A, Tsuchihashi K, Nagao K, Kyuma M, Ooiwa H, et al.Clinical implications of midventricular obstruction and intravenous propranolol use in transient left ventricular apical ballooning (Tako-tsubo cardiomyopathy). Am Heart J. 2008;155:1-7.

8. Bonacchi M, Maiani M, Harmelin G, Sani G. Intractable cardiogenic shock in stress cardiomyopathy with left ventricular outflow tract obstruction: is extra-corporeal life the best treatment? Eur J Heart Fail. 2009;11:721. 